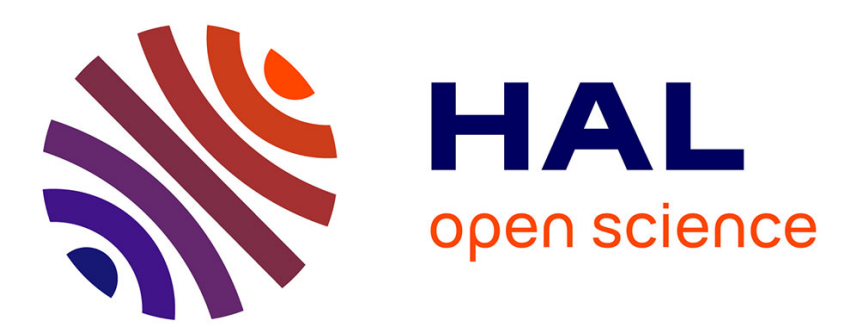

\title{
The motion of a fluid-rigid ball system at the zero limit of the rigid ball radius
}

Ana Leonor Silvestre, Takéo Takahashi

\section{To cite this version:}

Ana Leonor Silvestre, Takéo Takahashi. The motion of a fluid-rigid ball system at the zero limit of the rigid ball radius. Archive for Rational Mechanics and Analysis, 2014, 211 (3), pp.991-1012. 10.1007/s00205-013-0696-7 . hal-00914936

\section{HAL Id: hal-00914936 \\ https://hal.science/hal-00914936}

Submitted on 10 Dec 2013

HAL is a multi-disciplinary open access archive for the deposit and dissemination of scientific research documents, whether they are published or not. The documents may come from teaching and research institutions in France or abroad, or from public or private research centers.
L'archive ouverte pluridisciplinaire HAL, est destinée au dépôt et à la diffusion de documents scientifiques de niveau recherche, publiés ou non, émanant des établissements d'enseignement et de recherche français ou étrangers, des laboratoires publics ou privés. 


\title{
The motion of a fluid-rigid ball system at the zero limit of the rigid ball radius
}

\author{
Ana Leonor Silvestre, Takéo Takahashi
}

\begin{abstract}
We study the limiting motion of a system of a rigid ball moving in a Navier-Stokes fluid flow in $\mathbb{R}^{3}$ as the radius of the ball goes to zero. Recently, Dashti and Robinson solved this problem in the 2-D case, in the absence of rotation of the ball [Arch. Rational Mech. Anal. 200 (2011) 285-312]. This restriction was caused by the difficulty in obtaining appropriate uniform bounds on the second order derivatives of the fluid velocity when the rigid body can rotate. In this paper, we show how to obtain the required uniform bounds on the velocity fields in the 3-D case. These estimates then allow to pass to the zero limit of the ball radius and show that the solution of the coupled system converges to the solution of the Navier-Stokes equations describing the motion of only fluid in the whole space. The trajectory of the centre of the ball converges to a fluid particle trajectory, which justifies the use of rigid tracers for finding Lagrangian paths of fluid flow.
\end{abstract}

\section{Introduction}

Suppose that, relatively to an inertial reference frame, a homogeneous three-dimensional rigid body, represented by a closed ball $B_{r}(t):=\left\{y \in \mathbb{R}^{3}:|y-h(t)| \leqslant r\right\}$, is moving in a NavierStokes liquid which occupies the exterior domain $\Omega_{r}(t):=\mathbb{R}^{3} \backslash B_{r}(t)$. The function $h=h(t)$ describes the trajectory of the center of mass of the rigid body, which is also allowed to rotate, $\omega=\omega(t)$ being its angular velocity. Denoting by $v=v(t, y)$ the Eulerian velocity of the fluid and $q=q(t, y)$ the corresponding pressure in the same inertial frame, the evolution of the motion of the system body-liquid is described by the initial boundary value problem

$$
\begin{gathered}
\partial_{t} v(t, y)+\left(v(t, y) \cdot \nabla_{y}\right) v(t, y)=\nabla_{y} \cdot T(v(t, y), q(t, y)), \quad y \in \Omega_{r}(t), t \in(0, T), \\
\nabla_{y} \cdot v(t, y)=0, \quad y \in \Omega_{r}(t), t \in(0, T), \\
v(t, y)=h^{\prime}(t)+\omega(t) \times(y-h(t)) \quad y \in \partial B_{r}(t), t \in(0, T), \\
m_{r} h^{\prime \prime}(t)=-\int_{\partial B_{r}(t)} T(v(t, y), q(t, y)) n(t, y) \mathrm{d} S, \quad t \in(0, T), \\
J_{r} \omega^{\prime}(t)=-\int_{\partial B_{r}(t)}(y-h(t)) \times T(v(t, y), q(t, y)) n(t, y) \mathrm{d} S, \quad t \in(0, T), \\
v(0, y)=v^{0}(y), \quad y \in \Omega_{r}(0), \\
h(0)=h^{0}, \quad h^{\prime}(0)=h^{\prime 0}, \quad \omega(0)=\omega^{0} .
\end{gathered}
$$

In the above system, we have denoted by $n(t)=n(t, y)$ the external unit normal to $\Omega_{r}(t)$, and by $T(v, q)$ the stress tensor of the liquid, defined by

$$
\begin{gathered}
T(v, q)=2 \nu D(v)-q \mathrm{I}_{3} \\
D(v)_{i j}=\frac{1}{2}\left(\frac{\partial v_{i}}{\partial y_{j}}+\frac{\partial v_{j}}{\partial y_{i}}\right) \quad i, j=1,2,3
\end{gathered}
$$


where $\nu$ is the viscosity coefficient of the liquid and where $\mathrm{I}_{3}$ is the identity matrix of order 3 . Finally, $m_{r}$ and $J_{r}$ are respectively the mass of the body, and the moment or the matrix of inertia of the body. We have assumed that the density of the ball is constant and we denote it by $\rho$. Then $m_{r}$ and $J_{r}$ have explicit formulas:

$$
m_{r}=\frac{4}{3} \pi r^{3} \rho
$$

and

$$
J_{r}=\frac{8}{15} \pi r^{5} \rho \mathrm{I}_{3}
$$

Note that $J_{r}$ is independent of time since the structure is a homogeneous ball.

By means of the changes of variables

$$
\begin{aligned}
x & :=y-h(t), \\
u(t, x) & :=v(t, x+h(t)), \\
p(t, x) & :=q(t, x+h(t)), \\
\ell(t) & :=h^{\prime}(t),
\end{aligned}
$$

system (1)-(7) can be written in a frame attached to the rigid ball:

$$
\begin{gathered}
\partial_{t} u+((u-\ell) \cdot \nabla) u=\nabla \cdot T(u, p) \quad \text { in }(0, T) \times \Omega_{r}, \\
\nabla \cdot u=0 \quad \text { in }(0, T) \times \Omega_{r}, \\
u(t, x)=\ell(t)+\omega(t) \times x \quad \text { for } t \in(0, T), x \in \partial B_{r}, \\
m_{r} \ell^{\prime}=-\int_{\partial B_{r}} T(u, p) n \mathrm{~d} S \quad \text { in }(0, T), \\
J_{r} \omega^{\prime}=-\int_{\partial B_{r}} x \times T(u, p) n \mathrm{~d} S \quad \text { in }(0, T), \\
u(0, x)=u^{0}(x), \quad x \in \Omega_{r} \\
\ell(0)=\ell^{0}, \quad \omega(0)=\omega^{0} .
\end{gathered}
$$

The advantage of this formulation is that now the fluid domain is known and time-independent $\Omega_{r}=\left\{x \in \mathbb{R}^{3}:|x|>r\right\}$. As concerns the well-posedness of the system (13)-(19), we refer to the works of Galdi and Silvestre [9], Takahashi and Tucsnak [25]. In particular, for all $r$, for any $\left(u^{0}, \ell^{0}, \omega^{0}\right) \in H^{1}\left(\Omega_{r}\right) \times \mathbb{R}^{3} \times \mathbb{R}^{3}$ such that

$$
u^{0}(x)=\ell^{0}+\omega^{0} \times x \quad \text { on } \partial B_{r},
$$

the system (13)-(19) admits a unique local strong solution

$$
\begin{gathered}
u_{r} \in H^{1}\left(0, T ; L^{2}\left(\Omega_{r}\right)^{3}\right) \cap L^{\infty}\left(0, T ; H^{1}\left(\Omega_{r}\right)^{3}\right) \cap L^{2}\left(0, T ; H^{2}\left(\Omega_{r}\right)^{3}\right), \\
\ell_{r} \in H^{1}(0, T)^{3}, \quad \omega_{r} \in H^{1}(0, T)^{3} .
\end{gathered}
$$

Note that the maximal time of existence may be finite and may depend on $r$. In the last years, fluid-structure systems such as system (13)-(19) have been extensively studied. After the pioneer results of Judakov [18], Serre [22], and Weinberger [27], many results have been published in the case of exterior domain (see, for instance, $[23,3,11,10,8]$ ) or in the case of bounded domain (see, for instance, $[1,5,6,7,12,13,15]$ ).

As in the work of Dashti and Robinson [4], our aim is to investigate the limit of system (13)(19) when the radius $r$ of the ball goes to 0 . We show that the limiting system is the following Navier-Stokes system in $\mathbb{R}^{3}$ :

$$
\begin{gathered}
\partial_{t} u+((u-\ell) \cdot \nabla) u=\nabla \cdot T(u, p) \quad \text { in }(0, T) \times \mathbb{R}^{3}, \\
\nabla \cdot u=0 \quad \text { in }(0, T) \times \mathbb{R}^{3}, \\
u(0, x)=u^{0}(x), \quad x \in \mathbb{R}^{3} .
\end{gathered}
$$


The function $\ell$ is such that

$$
u(t, 0)=\ell(t) .
$$

In particular, using the change of variables (12) we conclude that

$$
\begin{gathered}
\partial_{t} v+(v \cdot \nabla) v=\nabla \cdot T(v, q) \quad \text { in }(0, T) \times \mathbb{R}^{3}, \\
\nabla \cdot v=0 \quad \text { in }(0, T) \times \mathbb{R}^{3}, \\
v(0, x)=v^{0}(x), \quad x \in \mathbb{R}^{3},
\end{gathered}
$$

and

$$
h^{\prime}(t)=v(t, h(t)) \quad \text { in }(0, T) .
$$

Dashti and Robinson [4] have solved this problem in $\mathbb{R}^{2}$, in the absence of rotation, considering $\omega=0$ by imposing an external torque and zero initial angular velocity of the disc. This last restriction has been made because of the difficulty in obtaining $L^{2}$-estimates independent of $r$ for $D^{2} u_{r}$ when the particle can rotate, see Lemma 4 of [4]. Such estimates are crucial to pass to the limit of the ball radius in the equations of motion of the fluid and show that the solution of the coupled system converges to the solution of the Navier-Stokes equations describing the motion of only fluid in the whole space.

The study of this type of asymptotic analysis problems is also considered in [16], [17] for the case of a fixed obstacle. In [21], a simplified model of combustion is analyzed with this approach.

In this paper we extend the results of Dashti and Robinson, considering the three-dimensional fluid-ball interaction problem (13)-(19). We show that the required uniform bounds on the velocity fields can be obtained by a careful analysis of a steady Stokes-type problem associated with the problem (13)-(19). Dashti and Robinson derived the estimates for $D^{2} u_{r}$ based on the classical exterior steady Stokes problem with a rigid velocity on the boundary. Here, we decompose the solution of the Stokes problem associated with the fluid-structure interaction problem in such a way that the explicit solutions for Stokes flow past a translating ball and around a rotating ball can be used to obtain a precise information about the dependence in $r$ when combined with the Newton's laws-type equations of the modified Stokes problem (see Theorem 3.1).

To state our main result more precisely, we extend the fluid velocities $u_{r}$ in $B_{r}$ by the rigid velocities:

$$
u_{r}(x)=\ell_{r}+\omega_{r} \times x \quad \text { in } B_{r} .
$$

This convention is used in all that follows. We also use some classical notation for functional spaces of divergence-free velocities $H$ and $V$. We define them in Section 2 (see (28), (29)).

Theorem 1.1. Assume $\left(u_{r}^{0}\right)_{0<r<r *}$ is a bounded sequence of $V$ with

$$
u_{r}^{0}(x)=\ell_{r}^{0}+\omega_{r}^{0} \times x \quad \text { in } B_{r},
$$

for all $r$ and assume that there exists $u^{0} \in V$ such that $u_{r}^{0} \rightarrow u^{0}$ in $H$ as $r \rightarrow 0$. Then there exists $T$ independent of $r$ such that for all $r$, the strong solution $\left(u_{r}, \ell_{r}, \omega_{r}\right)$ of (13)-(19) associated to $\left(u_{r}^{0}, \ell_{r}^{0}, \omega_{r}^{0}\right)$ exists on $(0, T)$. Moreover, for all $r_{0}, R>0$,

$$
\begin{gathered}
u_{r} \rightarrow u \quad \text { in } H^{1}\left(0, T ; L^{2}\left(\mathbb{R}^{3}\right)^{3}\right) \cap L^{2}\left(0, T ; H^{2}\left(\Omega_{r_{0}}\right)^{3}\right)-\text { weak, } \\
u_{r} \rightarrow u \quad \text { in } L^{\infty}\left(0, T ; H^{1}\left(\mathbb{R}^{3}\right)^{3}\right)-\text { weak*, } \\
u_{r} \rightarrow u \quad \text { strongly in } L^{2}\left(0, T ; L^{2}\left(B_{R}\right)^{3}\right), \\
\ell_{r} \rightarrow \ell \quad \text { strongly in } L^{2}(0, T)^{3},
\end{gathered}
$$

where $(u, \ell)$ is the solution of $(20)-(22)$, (23) associated to $u^{0}$.

The plan of the paper is the following. In Section 2, we introduce some notation and prove some auxiliary interpolation inequalities. Section 3 is devoted to the analysis of the above mentioned steady Stokes-type problem, where we obtain an uniform estimate in $r$ for the second derivatives of the solution of such a problem in terms of a norm of the associated Stokestype operator. In Section 4, we obtain a number of uniform estimates for the fluid-structure interaction problem (13)-(19) and, finally, in Section 5, we pass to the zero limit of the ball radius to obtain the Navier-Stokes flow (20)-(22). For the reader's convenience, we include in an Appendix (Section A) some calculations for the explicit Stokes solutions that have been used in Section 3. 


\section{Notation and auxiliary results}

For a sufficiently regular vector field $v: \mathbb{R}^{3} \rightarrow \mathbb{R}^{3}$, we denote by $\nabla v$ the second order tensor field whose components $(\nabla v)_{j k}$ are given by $\frac{\partial v_{k}}{\partial x_{j}}$, and by $D(v)$ we denote the symmetric part of $\nabla v$ (see (9)). The second order derivatives of $v$ will be indicated by $D^{2} v$, more precisely, for each $j \in\{1,2,3\}, D^{2} v_{j}$ is the Hessian matrix of $v_{j}$.

We shall use standard notation for function spaces. So, for instance, $L^{s}(\mathcal{D})$ and $H^{m}(\mathcal{D})$ will denote the usual Lebesgue and Sobolev spaces on the domain $\mathcal{D}$, with norms $\|\cdot\|_{s, \mathcal{D}}$ and $\|\cdot\|_{m, 2, \mathcal{D}}$, respectively. Notations like $L^{s}(\mathcal{D})^{3}\left(\operatorname{resp} . L^{s}(\mathcal{D})^{3 \times 3}\right), H^{m}(\mathcal{D})^{3}\left(\right.$ resp. $\left.H^{m}(\mathcal{D})^{3 \times 3}\right)$, etc. will be used to denote analogous spaces of vector-valued functions (resp. tensor-valued functions).

We also use the following classical notation

$$
\begin{gathered}
H:=\left\{\phi \in L^{2}\left(\mathbb{R}^{3}\right)^{3}: \nabla \cdot \phi=0 \text { in } \mathbb{R}^{3}\right\}, \\
V:=H \cap H^{1}\left(\mathbb{R}^{3}\right)^{3} .
\end{gathered}
$$

Finally, we consider in this paper the following subsets of $\mathbb{R}^{3}$ :

$$
\begin{gathered}
\Omega_{r}=\left\{x \in \mathbb{R}^{3}:|x|>r\right\}, \\
B_{r}=\left\{x \in \mathbb{R}^{3}:|x| \leqslant r\right\}, \\
B_{r, R}=\left\{x \in \mathbb{R}^{3}: R \geqslant|x|>r\right\},
\end{gathered}
$$

where $0<r<R$.

In the next lemma, we establish a useful interpolation inequality in the exterior domain $\Omega_{r}$, where the embedding constant is independent of $r$.

Lemma 2.1. Let $u \in H^{2}\left(\Omega_{r}\right)$. Then the following inequality holds

$$
\|u\|_{\infty, \Omega_{r}} \leqslant C\|u\|_{2, \Omega_{r}}^{1 / 4}\left\|D^{2} u\right\|_{2, \Omega_{r}}^{3 / 4}
$$

with a constant $C$ independent of $u$ and $r$.

Proof. Let $\mathcal{D} \subseteq \mathbb{R}^{3}$ be an exterior domain. In [2], Crispo and Maremonti proved, in particular, that any function $u \in L^{2}(\mathcal{D})$ with $D^{2} u \in L^{2}(\mathcal{D})^{3 \times 3}$ satisfies

$$
\|u\|_{\infty, \mathcal{D}} \leqslant C\left\|D^{2} u\right\|_{2, \mathcal{D}}^{\frac{3}{4}}\|u\|_{2, \mathcal{D}}^{\frac{1}{4}},
$$

where $C$ is a constant independent of $u$.

Let $u \in L^{2}\left(\Omega_{r}\right)$. Then, by a simple change of variables, we find the relation

$$
\|u\|_{2, \Omega_{r}}=r^{\frac{3}{2}}\left\|u^{r}\right\|_{2, \Omega_{1}}
$$

where $u^{r}(x):=u(r x)\left(x \in \Omega_{1}\right)$. Analogously, if the second derivatives of $u$ belong to $L^{2}\left(\Omega_{r}\right)$, we have

$$
\left\|D^{2} u\right\|_{2, \Omega_{r}}=r^{-\frac{1}{2}}\left\|D^{2} u^{r}\right\|_{2, \Omega_{1}} .
$$

Hence, applying (30) to $u^{r}$ and $\Omega_{1}$ yields

$$
\|u\|_{\infty, \Omega_{r}}=\left\|u^{r}\right\|_{\infty, \Omega_{1}} \leqslant C\left\|D^{2} u^{r}\right\|_{2, \Omega_{1}}^{\frac{3}{4}}\left\|u^{r}\right\|_{2, \Omega_{1}}^{\frac{1}{4}}
$$

with $C$ independent of $r$. But

$$
\left\|D^{2} u^{r}\right\|_{2, \Omega_{1}}^{\frac{3}{4}}=r^{\frac{3}{4}\left(-\frac{1}{2}\right)}\left\|D^{2} u\right\|_{2, \Omega_{r}}^{\frac{3}{4}}=r^{-\frac{3}{8}}\left\|D^{2} u\right\|_{2, \Omega_{r}}^{\frac{3}{4}}
$$

and

so that

$$
\left\|u^{r}\right\|_{2, \Omega_{1}}^{\frac{1}{4}}=r^{\frac{1}{4} \frac{3}{2}}\|u\|_{2, \Omega_{r}}^{\frac{1}{4}}=r^{\frac{3}{8}}\|u\|_{2, \Omega_{r}}^{\frac{1}{4}}
$$

$$
\|u\|_{\infty, \Omega} \leqslant C\left\|D^{2} u\right\|_{2, \Omega_{r}}^{\frac{3}{4}}\|u\|_{2, \Omega_{r}}^{\frac{1}{4}}
$$

with a constant $C$ independent of $r$. 
We have also the following estimate.

Lemma 2.2. Let $u \in H^{2}\left(\Omega_{r}\right)$. Then the following inequality holds

$$
\|u\|_{C^{0,1 / 2}\left(\Omega_{r}\right)} \leqslant C\left\|D^{2} u\right\|_{2, \Omega_{r}}
$$

with a constant $C$ independent of $u$ and $r$.

Proof. We proceed as in the proof of Lemma 2.1. More precisely, using [2] again, we know that for an exterior domain $\mathcal{D} \subseteq \mathbb{R}^{3}$, a function $u \in L^{2}(\mathcal{D})$ with $D^{2} u \in L^{2}(\mathcal{D})^{3 \times 3}$ satisfies

$$
\|u\|_{C^{0,1 / 2}(\mathcal{D})} \leqslant C\left\|D^{2} u\right\|_{2, \mathcal{D}}
$$

where $C$ is a constant independent of $u$. Then for $u \in L^{2}\left(\Omega_{r}\right)$ with $D^{2} u \in L^{2}\left(\Omega_{r}\right)^{3 \times 3}$, we set $u^{r}(x):=u(r x)\left(x \in \Omega_{1}\right)$, and we recall

$$
\left\|D^{2} u\right\|_{2, \Omega_{r}}=r^{-\frac{1}{2}}\left\|D^{2} u^{r}\right\|_{2, \Omega_{1}} .
$$

We can also prove

$$
\|u\|_{C^{0,1 / 2}\left(\Omega_{r}\right)}=r^{-1 / 2}\left\|u^{r}\right\|_{C^{0,1 / 2}\left(\Omega_{1}\right)} .
$$

Hence, applying (31) to $u^{r}$ and $\Omega_{1}$ yields the result.

As in Lemma 2 in [4], we can prove a useful result which shows how the trace inequality for the domain $\Omega_{r}$ depends on $r$ in the three-dimensional case.

Lemma 2.3. Let $w \in L_{l o c}^{1}\left(\overline{\Omega_{r}}\right)$ be such that $\nabla w \in L^{2}\left(\Omega_{r}\right)^{3}$. Then there exist constants $w_{0}$ and $C$ such that

$$
\left\|w-w_{0}\right\|_{L^{2}\left(\partial B_{r}\right)} \leqslant C r^{1 / 2}\|\nabla w\|_{L^{2}\left(\Omega_{r}\right)^{3}}
$$

where the constant $C$ is independent of $w$ and $r$. If $w \in L^{6}\left(\Omega_{r}\right)$ then $w_{0}=0$.

Proof. Let $w \in L_{l o c}^{1}\left(\overline{\Omega_{r}}\right)$ be such that $\nabla w \in L^{2}\left(\Omega_{r}\right)^{3}$ and set $w^{r}(y):=w(r y)\left(y \in \Omega_{1}\right)$. It is well known (see [2]) that there exist constants $w_{0}$ and $C$ such that

$$
\left\|w^{r}-w_{0}\right\|_{L^{6}\left(\Omega_{1}\right)} \leqslant C\left\|\nabla w^{r}\right\|_{L^{2}\left(\Omega_{1}\right)^{3}}
$$

where the constant $C$ is independent of $w$ and $r$, and $w_{0}=0$ if $w \in L^{6}\left(\Omega_{r}\right)$.

Now

$$
\int_{\partial B_{r}}\left|w-w_{0}\right|^{2} \mathrm{~d} S=r^{2} \int_{\partial B_{1}}\left|w^{r}-w_{0}\right|^{2} \mathrm{~d} S \leqslant C r^{2}\left[\int_{B_{1,2}}\left|w^{r}-w_{0}\right|^{2} \mathrm{~d} x+\int_{\Omega_{1}}\left|\nabla w^{r}\right|^{2} \mathrm{~d} x\right]
$$

and since

$$
\int_{B_{1,2}}\left|w^{r}-w_{0}\right|^{2} \mathrm{~d} x \leqslant C\left(\int_{B_{1,2}}\left|w^{r}-w_{0}\right|^{6} \mathrm{~d} x\right)^{1 / 3} \leqslant C\left\|w^{r}-w_{0}\right\|_{6, \Omega_{1}}^{2},
$$

from (32), we get

$$
\int_{\partial B_{r}}\left|w-w_{0}\right|^{2} \mathrm{~d} S \leqslant C r^{2}\left\|\nabla w^{r}\right\|_{2, \Omega_{1}}^{2}
$$

Finally, by changing variables again,

$$
\int_{\partial B_{r}}\left|w-w_{0}\right|^{2} \mathrm{~d} S \leqslant C r^{2}\left\|\nabla w^{r}\right\|_{2, \Omega_{1}}^{2} \leqslant C r\|\nabla w\|_{2, \Omega_{r}}^{2}
$$


Now we recall some function spaces appropriate for studying the system (13)-(19). The spaces

$$
\begin{aligned}
& \mathcal{H}_{r}:=\left\{\phi \in L^{2}\left(\mathbb{R}^{3}\right)^{3}: \nabla \cdot \phi=0 \text { in } \mathbb{R}^{3}, D(\phi)=0 \text { in } B_{r}\right\} \\
& \mathcal{V}_{r}:=\left\{\phi \in H^{1}\left(\mathbb{R}^{3}\right)^{3}: \nabla \cdot \phi=0 \text { in } \mathbb{R}^{3}, D(\phi)=0 \text { in } B_{r}\right\}
\end{aligned}
$$

are Hilbert spaces for the inner products of $L^{2}\left(\mathbb{R}^{3}\right)^{3}$ and $H^{1}\left(\mathbb{R}^{3}\right)^{3}$, respectively. It is well known that, for each $\phi \in \mathcal{H}_{r}$, there exist $\xi_{\phi} \in \mathbb{R}^{3}$ and $\omega_{\phi} \in \mathbb{R}^{3}$ such that

$$
\phi_{\mid B_{r}}(x)=\xi_{\phi}+\omega_{\phi} \times x .
$$

We also define a global density by

$$
\rho_{r}= \begin{cases}1 & \text { in } \Omega_{r}, \\ \rho & \text { in } B_{r},\end{cases}
$$

and the following inner product of $L^{2}\left(\mathbb{R}^{3}\right)^{3}$

$$
(u, v)_{\mathcal{H}_{r}}=\int_{\mathbb{R}^{3}} \rho_{r} u \cdot v d x .
$$

The corresponding norm is equivalent to the usual $L^{2}$ norm. The notation we choose for this inner product comes from the fact that if $u, v \in \mathcal{H}_{r}$, then

$$
(u, v)_{\mathcal{H}_{r}}=\int_{\Omega_{r}} u \cdot v d x+m_{r} \xi_{u} \cdot \xi_{v}+\left(J_{r} \omega_{u}\right) \cdot \omega_{v}
$$

It is also common (see [8]) to consider the space

$$
\left\{v \in C_{c}^{\infty}\left(\mathbb{R}^{3}\right)^{3}: \nabla \cdot v=0, D(v)=0 \text { in } B_{r}\right\}
$$

and its completion $\mathcal{W}_{r}$ with respect to the seminorm $\|D(v)\|_{2, \mathbb{R}^{3}}$. In what follows, we use several times the following classical relation

$$
\|\nabla v\|_{2, \mathbb{R}^{3}}^{2}=2\|D(v)\|_{2, \mathbb{R}^{3}}^{2}=2\|D(v)\|_{2, \Omega_{r}}^{2}, \forall v \in \mathcal{W}_{r}
$$

Let us recall (see [8, Lemma 4.11])

$$
\mathcal{W}_{r}=\left\{v \in L^{6}\left(\mathbb{R}^{3}\right)^{3}: \nabla v \in L^{2}\left(\mathbb{R}^{3}\right)^{3 \times 3}, \nabla \cdot v=0, D(v)=0 \text { in } B_{r}\right\} .
$$

We also recall the useful relations (see [8, Lemma 4.9])

$$
\begin{gathered}
r^{1 / 2}\left|\xi_{v}\right| \leqslant C\|\nabla v\|_{2, \mathbb{R}^{3}}, \forall v \in \mathcal{W}_{r}, \\
r^{3 / 2}\left|\omega_{v}\right| \leqslant C\|\nabla v\|_{2, \mathbb{R}^{3}}, \forall v \in \mathcal{W}_{r}
\end{gathered}
$$

for positive constants $C$ independent of $v$ and $r$.

Finally let us give the following result.

Lemma 2.4. Let $u \in \mathcal{V}_{r}$ be such that $u_{\mid \Omega_{r}} \in H^{2}\left(\Omega_{r}\right)^{3}$ and $u_{\mid B_{r}}(x)=\ell+\omega \times x$. Then

$$
|\ell| \leqslant\|u\|_{\infty, \Omega_{r}}
$$

Proof. We follow an idea of [4]. Since the normal to $\Omega_{r}$ is given by $n(x)=-\frac{x}{r}$, we have

$$
u \cdot n=\ell \cdot n \quad \text { on } \partial B_{r} .
$$

Now, by taking a point $\bar{x} \in \partial B_{r}$ such that $\ell \cdot n(\bar{x})=|\ell|$, we obtain

$$
|\ell|=|u(\bar{x}) \cdot n(\bar{x})| \leqslant|u(\bar{x})| \leqslant\|u\|_{\infty, \Omega_{r}} .
$$




\section{The Stokes-type problem and the operator $A_{r}$}

The semigroup approach of [25] for problem (13)-(19) is based on the operator defined by

$$
\begin{aligned}
& D\left(A_{r}\right):=\left\{\phi \in H^{1}\left(\mathbb{R}^{3}\right)^{3}: \phi_{\mid \Omega_{r}} \in H^{2}\left(\Omega_{r}\right)^{3}, \nabla \cdot \phi=0 \text { in } \mathbb{R}^{3}, D(\phi)=0 \text { in } B_{r}\right\} \\
& A_{r}:=\mathbb{P}_{r} \mathcal{A}_{r}
\end{aligned}
$$

with $\mathbb{P}_{r}$ the orthogonal projector in $L^{2}\left(\mathbb{R}^{3}\right)^{3}$ onto $\mathcal{H}_{r}$ (with respect to the inner product $(\cdot, \cdot) \mathcal{H}_{r}$ ) and

$$
\mathcal{A}_{r} u:= \begin{cases}-\nu \Delta u & \text { in } \Omega_{r} \\ \frac{2 \nu}{m_{r}} \int_{\partial B_{r}} D(u) n \mathrm{~d} S+\left[2 \nu J_{r}^{-1} \int_{\partial B_{r}} y \times D(u) n \mathrm{~d} S\right] \times x & \text { in } B_{r} .\end{cases}
$$

In this section, we analyze the operator $A_{r}$. Given $u \in D\left(A_{r}\right)$, there exist $\ell \in \mathbb{R}^{3}$ and $\omega \in \mathbb{R}^{3}$ such that

$$
u_{\mid B_{r}}(x)=\ell+\omega \times x .
$$

Let $f:=A_{r} u$. Since $f_{\mid \Omega_{r}} \in L^{2}\left(\Omega_{r}\right)^{3}$, there exists (see, for instance, [25]) $p \in L_{l o c}^{2}\left(\overline{\Omega_{r}}\right.$ ) with $\nabla p \in L^{2}\left(\Omega_{r}\right)^{3}$ such that

$$
\begin{gathered}
-\nabla \cdot T(u, p)=f_{\mid \Omega_{r}} \quad \text { in } \Omega_{r}, \\
\nabla \cdot u=0 \quad \text { in } \Omega_{r}, \\
u(x)=\ell+\omega \times x \quad \text { for } x \in \partial B_{r}, \\
\lim _{|x| \rightarrow \infty} u(x)=0, \\
m_{r} \ell_{f}=-\int_{\partial B_{r}} T(u, p) n \mathrm{~d} S, \\
J_{r} \omega_{f}=-\int_{\partial B_{r}} x \times T(u, p) n \mathrm{~d} S .
\end{gathered}
$$

Our aim is to show that

$$
\left\|D^{2} u\right\|_{2, \Omega_{r}} \leqslant C\left[\|\nabla u\|_{2, \mathbb{R}^{3}}+\left(\left\|f_{\mid \Omega_{r}}\right\|_{2, \Omega_{r}}^{2}+m_{r}\left|\ell_{f}\right|^{2}+J_{r} \omega_{f} \cdot \omega_{f}\right)^{\frac{1}{2}}\right]
$$

with $C$ independent of $u, f$ and $r$. Recall that $m_{r}=\mathcal{O}\left(r^{3}\right)$ and $J_{r}=\mathcal{O}\left(r^{5}\right)$.

Theorem 3.1. There exists a positive constant $C$ independent of $r$ such that

$$
\left\|D^{2} u\right\|_{2, \Omega_{r}} \leqslant C\left(\left\|A_{r} u\right\|_{\mathcal{H}_{r}}+\|\nabla u\|_{2, \mathbb{R}^{3}}\right) \quad\left(u \in D\left(A_{r}\right)\right) .
$$

Proof. We use the following decomposition

$$
(u, p)=\left(u^{(1)}, p^{(1)}\right)+\left(u^{(2)}, p^{(2)}\right)+\left(u^{(3)}, p^{(3)}\right)
$$

where $\left(u^{(1)}, p^{(1)}\right)$ satisfies

$$
\begin{gathered}
-\nabla \cdot T\left(u^{(1)}, p^{(1)}\right)=f_{\mid \Omega_{r}} \quad \text { in } \Omega_{r}, \\
\nabla \cdot u^{(1)}=0 \quad \text { in } \Omega_{r}, \\
u^{(1)}(x)=0 \quad \text { for } x \in \partial B_{r}, \\
\lim _{|x| \rightarrow \infty} u^{(1)}(x)=0
\end{gathered}
$$

and

$$
\begin{aligned}
\left(u^{(2)}(x), p^{(2)}(x)\right)= & \left(\frac{3 r}{4}\left[\frac{\ell}{|x|}+\frac{(\ell \cdot x) x}{|x|^{3}}\right]+\frac{r^{3}}{4}\left[\frac{\ell}{|x|^{3}}-3 \frac{(\ell \cdot x) x}{|x|^{5}}\right], \frac{3 \nu r}{2} \frac{\ell \cdot x}{|x|^{3}}\right) \\
& \left(u^{(3)}(x), p^{(3)}(x)\right)=\left(r^{3} \frac{\omega \times x}{|x|^{3}}, 0\right) .
\end{aligned}
$$


Using the results of Section A, we see that $\left(u^{(2)}, p^{(2)}\right)$ and $\left(u^{(3)}, p^{(3)}\right)$ satisfy

$$
\begin{gathered}
\nabla \cdot T\left(u^{(2)}, p^{(2)}\right)=0, \text { in } \Omega_{r}, \\
\nabla \cdot u^{(2)}=0, \text { in } \Omega_{r}, \\
u^{(2)}(x)=\ell, x \in \partial B_{r}, \\
\lim _{|x| \rightarrow \infty} u^{(2)}(x)=0, \\
\int_{\partial B_{r}} T\left(u^{(2)}, p^{(2)}\right) n d S=6 \pi \nu r \ell, \\
\int_{\partial B_{r}} x \times T\left(u^{(2)}, p^{(2)}\right) n d S=0
\end{gathered}
$$

and

$$
\begin{gathered}
\nabla \cdot T\left(u^{(3)}, p^{(3)}\right)=0, \text { in } \Omega_{r}, \\
\nabla \cdot u^{(3)}=0, \text { in } \Omega_{r}, \\
u^{(3)}(x)=\omega \times x, x \in \partial B_{r}, \\
\lim _{|x| \rightarrow \infty} u^{(3)}(x)=0, \\
\int_{\partial B_{r}} T\left(u^{(3)}, p^{(3)}\right) n d S=0, \\
\int_{\partial B_{r}} x \times T\left(u^{(3)}, p^{(3)}\right) n d S=8 \pi \nu r^{3} \omega .
\end{gathered}
$$

From (52), (53) and the results of Section A, we deduce the existence of a positive constant $C$ independent of $r$ such that

$$
\begin{aligned}
& \left\|D^{2} u^{(2)}\right\|_{2, \Omega_{r}} \leqslant C|\ell| r^{-1 / 2} \\
& \left\|D^{2} u^{(3)}\right\|_{2, \Omega_{r}} \leqslant C|\omega| r^{1 / 2} .
\end{aligned}
$$

Writing (44) and (45) in terms of the decomposition (47) of $(u, p)$ yields

$$
\begin{aligned}
& 6 \pi \nu r \ell=-m_{r} \ell_{f}-\int_{\partial B_{r}} T\left(u^{(1)}, p^{(1)}\right) n \mathrm{~d} S \\
& 8 \pi \nu r^{3} \omega=-J_{r} \omega_{f}-\int_{\partial B_{r}} x \times T\left(u^{(1)}, p^{(1)}\right) n \mathrm{~d} S .
\end{aligned}
$$

This implies

$$
\begin{gathered}
\ell=-\frac{2 r^{2}}{9 \nu} \ell_{f}-\frac{1}{6 \pi \nu r} \int_{\partial B_{r}} T\left(u^{(1)}, p^{(1)}\right) n \mathrm{~d} S \\
\omega=-\frac{r^{2}}{15 \nu} \omega_{f}-\frac{1}{8 \pi \nu r^{3}} \int_{\partial B_{r}} x \times T\left(u^{(1)}, p^{(1)}\right) n \mathrm{~d} S .
\end{gathered}
$$

By Lemma 1 of [14],

$$
\left\|D^{2} u^{(1)}\right\|_{2, \Omega_{r}} \leqslant C\left(\left\|f_{\mid \Omega_{r}}\right\|_{2, \Omega_{r}}+\left\|\nabla u^{(1)}\right\|_{2, \Omega_{r}}\right),
$$

with a constant $C$ independent of $r$. Moreover, we have

$$
\left\|\nabla u^{(1)}\right\|_{2, \Omega_{r}} \leqslant\|\nabla u\|_{2, \Omega_{r}}+\left\|\nabla u^{(2)}\right\|_{2, \Omega_{r}}+\left\|\nabla u^{(3)}\right\|_{2, \Omega_{r}} \leqslant\|\nabla u\|_{2, \Omega_{r}}+C|\ell| r^{1 / 2}+C|\omega| r^{3 / 2}
$$

and from (36) and (37) it follows

$$
\left\|\nabla u^{(1)}\right\|_{2, \Omega_{r}} \leqslant C\|\nabla u\|_{2, \mathbb{R}^{3}}
$$

so that

$$
\left\|D^{2} u^{(1)}\right\|_{2, \Omega_{r}} \leqslant C\left(\left\|f_{\mid \Omega_{r}}\right\|_{2, \Omega_{r}}+\|\nabla u\|_{2, \mathbb{R}^{3}}\right)
$$


In order to appropriatly bound $\ell$ and $\omega$ from (58) and (59), we begin by showing that the inequality

$$
\left|\int_{\partial B_{r}} x \times T\left(u^{(1)}, p^{(1)}\right) n \mathrm{~d} S\right| \leqslant C r^{5 / 2}\left\|f_{\mid \Omega_{r}}\right\|_{2, \Omega_{r}},
$$

holds with $C$ independent of $r$. Indeed, if we consider the functions $H_{i}(x):=r^{3} \frac{e_{i} \times x}{|x|^{3}}$, which belong to $L^{2}\left(\Omega_{r}\right)^{3}$, and note that for each $i=1,2,3$,

$$
e_{i} \cdot \int_{\partial B_{r}} x \times T\left(u^{(1)}, p^{(1)}\right) n \mathrm{~d} S=\int_{\partial B_{r}} e_{i} \times x \cdot T\left(u^{(1)}, p^{(1)}\right) n \mathrm{~d} S=\int_{\partial B_{r}} H_{i} \cdot T\left(u^{(1)}, p^{(1)}\right) n \mathrm{~d} S,
$$

by Green's formula for the Stokes equations, we have

$$
e_{i} \cdot \int_{\partial B_{r}} x \times T\left(u^{(1)}, p^{(1)}\right) n \mathrm{~d} S=\int_{\partial B_{r}} H_{i} \cdot T\left(u^{(1)}, p^{(1)}\right) n \mathrm{~d} S=-\int_{\Omega_{r}} f \cdot H_{i} \mathrm{~d} x .
$$

Then (62) follows from the fact that $\left\|H_{i}\right\|_{2} \leqslant \mathrm{Cr}^{5 / 2}$ (see Section A). It remains to show that

$$
\left|\int_{\partial B_{r}} T\left(u^{(1)}, p^{(1)}\right) n \mathrm{~d} S\right| \leqslant C r^{3 / 2}\left(\left\|f_{\mid \Omega_{r}}\right\|_{2, \Omega_{r}}+\|\nabla u\|_{2, \Omega_{r}}\right),
$$

where, again, $C$ is independent of $r$. Since

$$
\left|\int_{\partial B_{r}} T\left(u^{(1)}, p^{(1)}\right) n \mathrm{~d} S\right|=\left|\int_{\partial B_{r}} T\left(u^{(1)}, p^{(1)}-p_{0}\right) n \mathrm{~d} S\right| \leqslant C r\left(\left\|\nabla u^{(1)}\right\|_{2, \partial B_{r}}+\left\|p^{(1)}-p_{0}\right\|_{2, \partial B_{r}}\right)
$$

for any $p_{0} \in \mathbb{R}$, we can modify the pressure $p^{(1)}$ in such a way that, according to Lemma 2.3,

$$
\left\|p^{(1)}-p_{0}\right\|_{2, \partial B_{r}} \leqslant C r^{1 / 2}\left\|\nabla p^{(1)}\right\|_{2, \Omega_{r}} .
$$

Also by Lemma 2.3,

$$
\left\|\nabla u^{(1)}\right\|_{2, \partial B_{r}} \leqslant C r^{1 / 2}\left\|D^{2} u^{(1)}\right\|_{2, \Omega_{r}} .
$$

Now, we only have to note that

$$
\left\|\nabla p^{(1)}\right\|_{2, \Omega_{r}} \leqslant C\left(\|f\|_{2, \Omega_{r}}+\left\|D^{2} u^{(1)}\right\|_{2, \Omega_{r}}\right)
$$

and use (61) to bound $\left\|D^{2} u^{(1)}\right\|_{2, \Omega_{r}}$.

Finally, (58) and (59) combined with (63) and (62) yield

$$
\begin{gathered}
|\ell| \leqslant C r^{2}\left|\ell_{f}\right|+C r^{1 / 2}\left(\left\|f_{\mid \Omega_{r}}\right\|_{2, \Omega_{r}}+\|\nabla u\|_{2, \Omega_{r}}\right), \\
|\omega| \leqslant C r^{2}\left|\omega_{f}\right|+C \frac{1}{r^{1 / 2}}\left\|f_{\mid \Omega_{r}}\right\|_{2, \Omega_{r}},
\end{gathered}
$$

for constants $C$ independent of $r$. Coming back to (56) and (57) with the above estimates for $|\ell|$ and $|\omega|$, we get

$$
\begin{aligned}
& \left\|D^{2} u^{(2)}\right\|_{2, \Omega_{r}} \leqslant C r^{3 / 2}\left|\ell_{f}\right|+C\left(\left\|f_{\mid \Omega_{r}}\right\|_{2, \Omega_{r}}+\|\nabla u\|_{2, \mathbb{R}^{3}}\right) \\
& \left\|D^{2} u^{(3)}\right\|_{2, \Omega_{r}} \leqslant C r^{5 / 2}\left|\omega_{f}\right|+C\left\|f_{\mid \Omega_{r}}\right\|_{2, \Omega_{r}}
\end{aligned}
$$

which together with (61) and (47) yields

$$
\left\|D^{2} u\right\|_{2, \Omega_{r}} \leqslant C r^{3 / 2}\left|\ell_{f}\right|+C r^{5 / 2}\left|\omega_{f}\right|+C\left\|f_{\mid \Omega_{r}}\right\|_{2, \Omega_{r}}+C\|\nabla u\|_{2, \mathbb{R}^{3}} \leqslant C\left(\left\|A_{r} u\right\|_{\mathcal{H}_{r}}+\|\nabla u\|_{2, \mathbb{R}^{3}}\right) .
$$




\section{Estimates independent of $r$ for the solution of (13)-} (19)

Assume that $u_{r}^{0} \in \mathcal{V}_{r}$ for some $r>0$. Then, writing $u_{r}^{0}=\ell_{r}^{0}+\omega_{r}^{0} \times x$ in $B_{r}$, and taking $\left(u_{r}^{0}, \ell_{r}^{0}, \omega_{r}^{0}\right)$ for the initial conditions of (13)-(19), we obtain (see, for instance, [25]) the existence and uniqueness of a strong solution

$$
u_{r} \in H^{1}\left(0, T ; L^{2}\left(\mathbb{R}^{3}\right)^{3}\right) \cap L^{\infty}\left(0, T ; H^{1}\left(\mathbb{R}^{3}\right)^{3}\right) \cap L^{2}\left(0, T ; H^{2}\left(\Omega_{r}\right)^{3}\right) .
$$

Here and in what follows, we extend $u_{r}$ to $B_{r}$ by $\ell_{r}+\omega_{r} \times x$. Let us note that the maximal time of existence $T$ may be finite and may depend on $r$.

We can write (13)-(17) in an abstract way by using the operator $A_{r}$ defined by (38)-(39) and the following nonlinear operator:

$$
\mathcal{N}_{r}(u)=\left\{\begin{array}{ll}
-((u-\ell) \cdot \nabla) u & \text { in } \Omega_{r} \\
0 & \text { in } B_{r}
\end{array} \quad\left(u \in \mathcal{V}_{r}\right),\right.
$$

and its projection in $\mathcal{H}_{r}$

$$
N_{r}(u):=\mathbb{P}_{r} \mathcal{N}_{r}(u)
$$

With this notation, we can write (13)-(17) as

$$
\partial_{t} u_{r}+A_{r} u_{r}=N_{r}\left(u_{r}\right) .
$$

Theorem 4.1. Assume $\left(u_{r}^{0}\right)_{0<r<r^{*}}$ is bounded in $H^{1}\left(\mathbb{R}^{3}\right)^{3}, u_{r}^{0} \in \mathcal{V}_{r}$ for all $r$. Then there exists $T$ independent of $r$ such that

(i) $\left(u_{r}\right)_{0<r<r^{*}}$ is bounded in $H^{1}\left(0, T ; L^{2}\left(\mathbb{R}^{3}\right)^{3}\right) \cap L^{\infty}\left(0, T ; H^{1}\left(\mathbb{R}^{3}\right)^{3}\right) \cap L^{2}\left(0, T ; H^{2}\left(\Omega_{r}\right)^{3}\right)$,

(ii) $\left(\ell_{r}\right)_{0<r<r^{*}}$ is bounded in $L^{2}(0, T)^{3}$.

Proof. Let us multiply (64) by $u_{r}$ and integrate over $\Omega_{r}$. We obtain

$$
\frac{1}{2} \frac{d}{d t}\left\|u_{r}\right\|_{\mathcal{H}_{r}}^{2}+2 \nu\left\|D\left(u_{r}\right)\right\|_{2, \Omega_{r}}^{2}=0
$$

which, combined with (35) yields

$$
\sup _{t \in(0, T)}\left\|u_{r}(t)\right\|_{2, \mathbb{R}^{3}}^{2}+\nu \int_{0}^{T}\left\|\nabla u_{r}\right\|_{2, \mathbb{R}^{3}}^{2} d t \leqslant C\left\|u_{r}^{0}\right\|_{2, \mathbb{R}^{3}}^{2} .
$$

Now we multiply (64) by $A_{r} u_{r}$ and integrate by parts over $\Omega_{r}$ to get

$$
\nu \frac{d}{d t}\left\|D\left(u_{r}\right)\right\|_{2, \Omega_{r}}^{2}+\left\|A_{r} u_{r}\right\|_{\mathcal{H}_{r}}^{2}=\left(N_{r}\left(u_{r}\right), A_{r} u_{r}\right)_{\mathcal{H}_{r}},
$$

where

$$
\left(N_{r}\left(u_{r}\right), A_{r} u_{r}\right)_{\mathcal{H}_{r}} \leqslant\left\|N_{r}\left(u_{r}\right)\right\|_{\mathcal{H}_{r}}\left\|A_{r} u_{r}\right\|_{\mathcal{H}_{r}} \leqslant\left\|\left(\left(u_{r}-\ell_{r}\right) \cdot \nabla\right) u_{r}\right\|_{2, \Omega_{r}}\left\|A_{r} u_{r}\right\|_{\mathcal{H}_{r}} .
$$

By Hölder's inequality,

$$
\left\|\left(u_{r} \cdot \nabla\right) u_{r}\right\|_{2, \Omega_{r}} \leqslant\left\|u_{r}\right\|_{\infty, \Omega_{r}}\left\|\nabla u_{r}\right\|_{2, \Omega_{r}}
$$

and by Lemma 2.4

$$
\left\|\left(\ell_{r} \cdot \nabla\right) u_{r}\right\|_{2, \Omega_{r}} \leqslant\left\|u_{r}\right\|_{\infty, \Omega_{r}}\left\|\nabla u_{r}\right\|_{2, \Omega_{r}} .
$$

Again we use (69), (70) and Lemma 2.1 to get

$$
\begin{aligned}
\left\|\left(\left(u_{r}-\ell_{r}\right) \cdot \nabla\right) u_{r}\right\|_{2, \Omega_{r}} & \leqslant 2\left\|u_{r}\right\|_{\infty, \Omega_{r}}\left\|\nabla u_{r}\right\|_{2, \Omega_{r}} \\
& \leqslant C\left\|u_{r}\right\|_{2, \Omega_{r}}^{1 / 4}\left\|D^{2} u_{r}\right\|_{2, \Omega_{r}}^{3 / 4}\left\|\nabla u_{r}\right\|_{2, \Omega_{r}}
\end{aligned}
$$

with a constant $C$ independent of $r$. By combining (71) with (67) and (68), we deduce that

$$
\nu \frac{d}{d t}\left\|D\left(u_{r}\right)\right\|_{2, \Omega_{r}}^{2}+\left\|A_{r} u_{r}\right\|_{\mathcal{H}_{r}}^{2} \leqslant C\left\|u_{r}\right\|_{2, \Omega_{r}}^{1 / 4}\left\|D^{2} u_{r}\right\|_{2, \Omega_{r}}^{3 / 4}\left\|\nabla u_{r}\right\|_{2, \Omega_{r}}\left\|A_{r} u_{r}\right\|_{\mathcal{H}_{r}} .
$$


Now, using Theorem 3.1, and (35) implies

$\frac{\nu}{2} \frac{d}{d t}\left\|\nabla u_{r}\right\|_{2, \mathbb{R}^{3}}^{2}+\left\|A_{r} u_{r}\right\|_{\mathcal{H}_{r}}^{2} \leqslant C\left\|u_{r}\right\|_{2, \Omega_{r}}^{1 / 4}\left\|\nabla u_{r}\right\|_{2, \Omega_{r}}\left\|A_{r} u_{r}\right\|_{\mathcal{H}_{r}}^{7 / 4}+C\left\|u_{r}\right\|_{2, \Omega_{r}}^{1 / 4}\left\|\nabla u_{r}\right\|_{2, \Omega_{r}}^{7 / 4}\left\|A_{r} u_{r}\right\|_{\mathcal{H}_{r}}$.

Applying Young's inequality to the above estimate yields

$$
\nu \frac{d}{d t}\left\|\nabla u_{r}\right\|_{2, \mathbb{R}^{3}}^{2}+\left\|A_{r} u_{r}\right\|_{\mathcal{H}_{r}}^{2} \leqslant C\left(\left\|u_{r}\right\|_{2, \Omega_{r}}^{2}+1\right)\left\|\nabla u_{r}\right\|_{2, \Omega_{r}}^{8}+C\left\|u_{r}\right\|_{2, \Omega_{r}}^{8 / 9}
$$

with $C$ independent of $r$. Using the estimate (66) we get

$$
\nu \frac{d}{d t}\left\|\nabla u_{r}\right\|_{2, \mathbb{R}^{3}}^{2}+\left\|A_{r} u_{r}\right\|_{\mathcal{H}_{r}}^{2} \leqslant C\left(1+\left\|u_{r}^{0}\right\|_{2, \mathbb{R}^{3}}^{8 / 9}+\left\|u_{r}^{0}\right\|_{2, \mathbb{R}^{3}}^{2}\right)\left(\left\|\nabla u_{r}\right\|_{2, \mathbb{R}^{3}}^{2}+1\right)^{4}
$$

Let $y(t):=1+\left\|\nabla u_{r}(t)\right\|_{2, \mathbb{R}^{3}}^{2}$. Then, from (73) we have

$$
y^{\prime} \leqslant C y^{4}
$$

with $C$ independent of $r$. We conclude that

$$
y(t) \leqslant \frac{y(0)}{\sqrt[3]{1-3 y(0)^{3} C t}}
$$

as long as $t<1 /\left(3 y(0)^{3} C\right)$. Let us consider $T$ small enough so that

$$
T<\frac{1}{3 C\left(1+\left\|\nabla u_{r}^{0}\right\|_{2, \mathbb{R}^{3}}^{2}\right)^{3}} .
$$

Since $\left(u_{r}^{0}\right)_{0<r<r *}$ is bounded in $H^{1}\left(\mathbb{R}^{3}\right)^{3}, T$ can be chosen so that it is independent of $r$.

We deduce that for all $t \in[0, T]$,

$$
\left\|\nabla u_{r}(t)\right\|_{2, \mathbb{R}^{3}}^{2} \leqslant C\left(1+\left\|\nabla u_{r}(0)\right\|_{2, \mathbb{R}^{3}}^{2}\right) \leqslant \tilde{K}_{1}
$$

with $\tilde{K}_{1}$ independent of $r$. Now from (73),

$$
\int_{0}^{T}\left\|A_{r} u_{r}(s)\right\|_{\mathcal{H}_{r}}^{2} d s \leqslant \tilde{K}_{2}
$$

with $\tilde{K}_{2}$ independent of $r$, which implies with Theorem 3.1

$$
\left\|D^{2} u_{r}\right\|_{L^{2}\left(0, T ; L^{2}\left(\Omega_{r}\right)^{3 \times 3 \times 3)}\right.} \leqslant \tilde{K}_{3}
$$

with $\tilde{K}_{3}$ independent of $r$. We can find constants $\tilde{K}_{4}$ and $\tilde{K}_{5}$ independent of $r$ such that

$$
\left\|\ell_{r}\right\|_{L^{2}\left(0, T ; \mathbb{R}^{3}\right)} \leqslant \tilde{K}_{4}
$$

and

$$
\left\|\partial_{t} u_{r}\right\|_{L^{2}\left(0, T ; L^{2}\left(\Omega_{r}\right)^{3}\right)} \leqslant \tilde{K}_{5}
$$

\section{Passage to the zero limit of the ball radius in (13)-} (19)

The aim of this section is to prove the main result of the paper.

Applying Theorem 4.1 and using a diagonal process, we deduce that for all $r_{0}>0$,

$$
\begin{gathered}
u_{r} \rightarrow u \quad \text { in } H^{1}\left(0, T ; L^{2}\left(\mathbb{R}^{3}\right)^{3}\right) \cap L^{2}\left(0, T ; H^{2}\left(\Omega_{r_{0}}\right)^{3}\right)-\text { weak } \\
u_{r} \rightarrow u \quad \text { in } L^{\infty}\left(0, T ; H^{1}\left(\mathbb{R}^{3}\right)^{3}\right)-\text { weak }^{*} \\
\ell_{r} \rightarrow \ell \quad \text { in } L^{2}(0, T)-\text { weak. }
\end{gathered}
$$


In particular for any $r_{0}, u \in L^{2}\left(0, T ; H^{2}\left(\Omega_{r_{0}}\right)^{3}\right)$ and its norm in these spaces is bounded independently of $r_{0}$. Following the proof of [4], we deduce that $u \in L^{2}\left(0, T ; H^{2}\left(\mathbb{R}^{3}\right)^{3}\right)$. Applying AubinLions-Simon Lemma, and using the fact that $\left(u_{r}\right)_{0<r<r^{*}}$ is bounded in $L^{2}\left(0, T ; H^{1}\left(B_{R}\right)^{3}\right) \cap$ $H^{1}\left(0, T ; L^{2}\left(B_{R}\right)^{3}\right)$, we conclude that there exists a subsequence such that

$$
u_{r} \rightarrow u \text { strongly in } L^{2}\left(0, T ; L^{2}\left(B_{R}\right)^{3}\right) \text {. }
$$

In order to pass to the limit as $r \rightarrow 0$, we write an integral formulation of (13)-(17). Assume $\varphi \in C^{1}\left(\mathbb{R}^{3}\right)^{3}$ is divergence free, with compact support in $B_{s, R}, R>s>0$. Then for $r<s$, we multiply (64) by $\varphi$ to obtain

$$
\int_{\mathbb{R}^{3}} \rho_{r} \partial_{t} u_{r} \cdot \varphi d x+\left(A_{r} u_{r}, \varphi\right)_{\mathcal{H}_{r}}=\left(N_{r}\left(u_{r}\right), \varphi\right)_{\mathcal{H}_{r}} \quad \text { in }(0, T) .
$$

By integrating by parts, we deduce from the above equation that

$$
\left.\int_{\mathbb{R}^{3}}\left(\partial_{t} u_{r}-\nu \Delta u_{r}\right) \cdot \varphi d x-\int_{\mathbb{R}^{3}}\left[\left(u_{r}-\ell_{r}\right) \cdot \nabla\right) \varphi\right] \cdot u_{r} d x=0 \quad \text { in }(0, T) .
$$

Using (74)-(76) and (77), we deduce that for all $\varphi \in C^{1}\left(\mathbb{R}^{3}\right)^{3}$ divergence free, with compact support in $B_{s, R}, R>s>0$,

$$
\int_{\mathbb{R}^{3}}\left(\partial_{t} u-\nu \Delta u+((u-\ell) \cdot \nabla) u\right) \cdot \varphi d x=0 \quad \text { in }(0, T) .
$$

We observe that the functions in $C^{1}\left(\mathbb{R}^{3}\right)^{3}$ which are divergence free, with compact support in $B_{s, R}, R>s>0$ are dense in $H$. To be complete we give below a proof of this result based on [20, pp. 165-166]. Consequently, we deduce that (79) holds for all $\varphi \in L^{2}(0, T ; H)$. In particular, (see, for instance, [26, pp. 14-15]), there exists $p$ with $\nabla p \in L^{2}\left(0, T ; L^{2}\left(\mathbb{R}^{3}\right)\right)$ such that

$$
\partial_{t} u-\nu \Delta u+((u-\ell) \cdot \nabla) u=-\nabla p \quad \text { in }(0, T) \times \mathbb{R}^{3} .
$$

To recover the initial condition, we apply a classical method: using (74) and (77), we deduce that for any function $\varphi \in C^{1}\left([0, T) \times \mathbb{R}^{3}\right)^{3}$ with compact support, we have

$$
\begin{aligned}
\int_{\mathbb{R}^{3}} u_{r}(0) \cdot \varphi(0) d x & =\int_{(0, T) \times \mathbb{R}^{3}} u_{r} \cdot \partial_{t} \varphi d t d x-\int_{(0, T) \times \mathbb{R}^{3}} \partial_{t} u_{r} \cdot \varphi d t d x \\
& \rightarrow \int_{(0, T) \times \mathbb{R}^{3}} u \cdot \partial_{t} \varphi d t d x-\int_{(0, T) \times \mathbb{R}^{3}} \partial_{t} u \cdot \varphi d t d x=\int_{\mathbb{R}^{3}} u(0) \cdot \varphi(0) d x .
\end{aligned}
$$

Since $u_{r}^{0} \rightarrow u^{0}$ weakly in $L^{2}\left(\mathbb{R}^{3}\right)^{3}$, then we deduce that $u(0)=u^{0}$.

Let us now prove the strong convergence of $\left(\ell_{r}\right)_{0<r<r^{*}}$. Let us consider a decreasing sequence $r_{k} \rightarrow 0$. Then, for $k>m$ and for any $n \in \partial B_{1}$,

$$
\begin{aligned}
\left|\left(\ell_{r_{k}}-\ell_{r_{m}}\right) \cdot n\right| & =\left|\left(u_{r_{k}}\left(r_{k}(-n)\right)-u_{r_{m}}\left(r_{m}(-n)\right)\right) \cdot n\right| \\
& \leqslant\left|\left(u_{r_{k}}\left(r_{m}(-n)\right)-u_{r_{m}}\left(r_{m}(-n)\right)\right) \cdot n\right|+\left|\left(u_{r_{k}}\left(r_{k}(-n)\right)-u_{r_{k}}\left(r_{m}(-n)\right)\right) \cdot n\right| \\
& \leqslant|| u_{r_{k}}-u_{r_{m}}\left\|_{\infty, B_{r_{m}, 2 r_{m}}}+\left|r_{k}-r_{m}\right|^{1 / 2}\right\| u_{r_{k}} \|_{C^{0,1 / 2}\left(\Omega_{r_{k}}\right)} .
\end{aligned}
$$

Let us note that, with an argument similar to the proof of Lemma 2.1, we can prove that for $w \in H^{2}\left(\Omega_{r}\right)$ the following inequality holds

$$
\|w\|_{\infty, B_{r, 2 r}} \leqslant C\|w\|_{2, B_{r, 2 r}}^{1 / 4}\left\|D^{2} w\right\|_{2, B_{r, 2 r}}^{3 / 4}
$$

with a constant $C>0$ independent of $w$ and $r$.

Combining (80) with the above result, Lemma 2.2 and with Theorem 4.1, we conclude that $\ell_{r} \rightarrow \ell$ in $L^{2}(0, T)^{3}$. To obtain the relation between $\ell$ and $u$, we consider a similar calculation: for a fixed $e \in \partial B_{1}$,

$$
|u(t, 0)-\ell(t)| \leqslant|u(t, 0)-u(t, r e)|+\left|u(t, r e)-\ell_{r}(t)\right|+\left|\ell_{r}(t)-\ell(t)\right| .
$$


The first term of the right-hand side of (81) can be estimated by $\|u\|_{C^{0,1 / 2}\left(\mathbb{R}^{3}\right)}$, the last term has just been treated, and the second term can be handle through the following manipulation: for any $n, e \in \partial B_{1}$,

$$
\begin{aligned}
\left|\left(u(t, r e)-\ell_{r}(t)\right) \cdot n\right| & =\left|\left(u(t, r e)-u_{r}(t,-r n)\right) \cdot n\right| \\
& \leqslant|(u(t, r e)-u(t,-r n)) \cdot n|+\left|\left(u(t,-r n)-u_{r}(t,-r n)\right) \cdot n\right| \\
& \leqslant r^{1 / 2}\|u\|_{C^{0,1 / 2}\left(\mathbb{R}^{3}\right)}+\left\|u-u_{r}\right\|_{\infty, B_{r, 2 r}}
\end{aligned}
$$

and we conclude as above.

This concludes the proof of Theorem 1.1.

We give here the proof of the density in $H$ of the subspace of the functions $C^{1}\left(\mathbb{R}^{3}\right)^{3}$ which are divergence free, with compact support in $B_{s, R}, R>s>0$. The proof is based on [20, pp. 165-166].

Lemma 5.1. Given $u \in H$ and $\delta>0$, there exist $0<s<1$ and $u^{s} \in V$ with supp $u^{s} \subset B_{s, 1 / s}$ such that

$$
\left\|u-u^{s}\right\|_{2, \mathbb{R}^{3}}<\delta .
$$

Proof. Given $u \in H$ and $\delta>0$, using the density of $V$ in $H$, there exists $v \in V$ such that

$$
\|u-v\|_{2, \mathbb{R}^{3}}<\frac{\delta}{2} .
$$

For $0<\varepsilon<1$ and $v \in V$, consider the problem of finding $v_{\varepsilon} \in V$ such that

$$
\begin{aligned}
& \Delta v_{\varepsilon}-v_{\varepsilon}-\nabla p_{\varepsilon}=\Delta v-v \text { in } B_{\varepsilon, 1 / \varepsilon}, \\
& \nabla \cdot v_{\varepsilon}=0 \text { in } B_{\varepsilon, 1 / \varepsilon} .
\end{aligned}
$$

This problem has a unique solution $\left(v_{\varepsilon}, p_{\varepsilon}\right) \in V \times L^{2}\left(B_{\varepsilon, 1 / \varepsilon}\right) / \mathbb{R}$ such that

$$
\left\|v_{\varepsilon}\right\|_{2, B_{\varepsilon, 1 / \varepsilon}}+\left\|\nabla v_{\varepsilon}\right\|_{2, B_{\varepsilon, 1 / \varepsilon}} \leqslant\|v\|_{V}
$$

Moreover, $p_{\varepsilon}$ can be normalized in such a way that

$$
\left\|p_{\varepsilon}\right\|_{2, B_{\varepsilon, 1 / \varepsilon}} \leqslant C\|v\|_{V}
$$

with $C$ independent of $\varepsilon$ and $v$. From (83), extending each $v_{\varepsilon}$ by zero outside $B_{\varepsilon, 1 / \varepsilon}$, we deduce the existence of $\bar{v} \in V$ such that

$$
v_{\varepsilon} \rightarrow \bar{v} \text { weakly in } V, \text { as } \varepsilon \rightarrow 0,
$$

while (84) implies the existence of $p \in L^{2}\left(\mathbb{R}^{3}\right)$ such that

$$
p_{\varepsilon} \rightarrow p \text { weakly in } L^{2}\left(\mathbb{R}^{3}\right) \text {, as } \varepsilon \rightarrow 0 .
$$

The function $\bar{v}-v \in V$ satisfies

$$
\begin{aligned}
& \Delta(\bar{v}-v)-(\bar{v}-v)-\nabla p=0 \text { in } \mathbb{R}^{3}, \\
& \nabla \cdot(\bar{v}-v)=0 \text { in } \mathbb{R}^{3}
\end{aligned}
$$

and therefore $\bar{v}=v$. Actually, since $v_{\varepsilon} \rightarrow v$ weakly in $V$ and $\left\|v_{\varepsilon}\right\|_{V} \leqslant\|v\|_{V}$, it follows that

$$
v_{\varepsilon} \rightarrow v \text { strongly in } V \text {, as } \varepsilon \rightarrow 0 .
$$

This results allows us to take $v_{s} \in V$ with support in $B_{s, 1 / s}$ such that

$$
\left\|v-v_{s}\right\|_{2, \mathbb{R}^{3}}<\frac{\delta}{2}
$$




\section{A Computations involving the auxiliary Stokes solu- tions}

Stokes derived in 1851 [24] (see also [19]) an explicit solution for tridimensional Stokes flow past a translating sphere. Specifically, the solution to the Stokes problem

$$
\begin{gathered}
\nabla \cdot T(u, p)=0, \text { in } \Omega_{r}, \\
\nabla \cdot u=0, \text { in } \Omega_{r}, \\
u(x)=\xi \text { for } x \in \partial B_{r}, \\
\lim _{|x| \rightarrow \infty} u(x)=0
\end{gathered}
$$

where $\xi \in \mathbb{R}^{3}$, is given by

$$
\begin{gathered}
u(x)=\frac{3 r}{4}\left[\frac{\xi}{|x|}+\frac{(\xi \cdot x) x}{|x|^{3}}\right]+\frac{r^{3}}{4}\left[\frac{\xi}{|x|^{3}}-3 \frac{(\xi \cdot x) x}{|x|^{5}}\right] \\
p(x)=\frac{3 \nu r}{2} \frac{\xi \cdot x}{|x|^{3}}+c
\end{gathered}
$$

where $c$ is a constant. By direct calculations, we find that the gradient of $u$ is

$$
\begin{aligned}
\nabla u(x)=\frac{3 r}{4}\left[\frac{\xi \otimes x-x \otimes \xi}{|x|^{3}}+\frac{(\xi \cdot x)}{|x|^{3}} I_{3}\right. & \left.-\frac{3(\xi \cdot x)}{|x|^{5}} x \otimes x\right] \\
+ & \frac{r^{3}}{4}\left[-3 \frac{(\xi \cdot x) I_{3}+\xi \otimes x+x \otimes \xi}{|x|^{5}}+\frac{15(\xi \cdot x) x \otimes x}{|x|^{7}}\right]
\end{aligned}
$$

and its symmetric part is

$$
D u(x)=\frac{3 r}{4}\left[\frac{(\xi \cdot x)}{|x|^{3}} I_{3}-\frac{3(\xi \cdot x)}{|x|^{5}} x \otimes x\right]+\frac{r^{3}}{4}\left[-3 \frac{(\xi \cdot x) I_{3}+\xi \otimes x+x \otimes \xi}{|x|^{5}}+\frac{15(\xi \cdot x) x \otimes x}{|x|^{7}}\right] .
$$

Therefore, since the external unit normal to $\Omega_{r}$ is given by $n(x)=-\frac{x}{r}$ and

$$
(\xi \otimes x) n(x)=-r \xi, \quad(x \otimes \xi) n(x)=(\xi \cdot x) n(x), \quad(x \otimes x) n(x)=r^{2} n(x) \quad\left(x \in \partial B_{r}\right)
$$

we have

$$
\begin{aligned}
T(u, p) n & =\frac{3}{2 r} \xi \text { on } \partial B_{r} \\
\int_{\partial B_{r}} T(u, p) n d S & =\frac{3}{2 r} \xi \int_{\partial B_{r}} d S=6 \pi r \xi \\
\int_{\partial B_{r}} x \times T(u, p) n d S & =-\frac{3}{2 r} \xi \times \int_{\partial B_{r}} x d S=0 .
\end{aligned}
$$

As concerns the summability properties of $u$ and its derivatives, we have

$$
\begin{aligned}
& u \in L^{q}\left(\Omega_{r}\right)^{3}, \forall q>3, \text { with }\|u\|_{q, \Omega_{r}} \leqslant C(q)|\xi| r^{3 / q}, \\
& \nabla u \in L^{q}\left(\Omega_{r}\right)^{3 \times 3}, \forall q>3 / 2 \text {, with }\|\nabla u\|_{q, \Omega_{r}} \leqslant C(q)|\xi| r^{3 / q-1} \\
& D^{2} u \in L^{q}\left(\Omega_{r}\right)^{3 \times 3 \times 3}, \forall q>1 \text {, with }\left\|D^{2} u\right\|_{q, \Omega_{r}} \leqslant C(q)|\xi| r^{3 / q-2} .
\end{aligned}
$$

In particular, $\|\nabla u\|_{2, \Omega_{r}} \leqslant C|\xi| r^{1 / 2}$ and $\left\|D^{2} u\right\|_{2, \Omega_{r}} \leqslant C|\xi| r^{-1 / 2}$.

Now we consider the Stokes flow around a rotating ball. The solution to the Stokes problem

$$
\begin{gathered}
\nabla \cdot T(u, p)=0, \text { in } \Omega_{r}, \\
\nabla \cdot u=0, \text { in } \Omega_{r}, \\
u(x)=\omega \times x \text { for } x \in \partial B_{r}, \\
\lim _{|x| \rightarrow \infty} u(x)=0
\end{gathered}
$$


where $\omega \in \mathbb{R}^{3}$, is (see $[24,19]$ )

$$
\begin{gathered}
u(x)=r^{3} \frac{\omega \times x}{|x|^{3}} \\
p(x)=c
\end{gathered}
$$

where $c$ is a constant. The gradient of $u$ and its symmetric part are given by

$$
\nabla u(x)=\frac{r^{3}}{|x|^{3}} W-\frac{3 r^{3}(\omega \times x) \otimes x}{|x|^{5}} \text { with } W=\left[\begin{array}{ccc}
0 & \omega_{3} & -\omega_{2} \\
-\omega_{3} & 0 & \omega_{1} \\
\omega_{2} & -\omega_{1} & 0
\end{array}\right]
$$

and

$$
D u(x)=-\frac{3 r^{3}}{2|x|^{5}}[x \otimes(\omega \times x)+(\omega \times x) \otimes x]
$$

We have

$$
\begin{aligned}
& D u(x) n(x)=\frac{3 \omega \times x}{2 r}, \\
& x \times D u(x) n(x)=\frac{3 x \times(\omega \times x)}{2 r} \quad\left(x \in \partial B_{r}\right)
\end{aligned}
$$

and then, due to the symmetry of the domain $\Omega_{r}$,

$$
\begin{gathered}
\int_{\partial B_{r}} T(u, p) n \mathrm{~d} S=0, \\
\int_{\partial B_{r}} x \times T(u, p) n \mathrm{~d} S=2 \nu \int_{\partial B_{r}} x \times(D u(x) n(x)) \mathrm{d} S=\frac{3 \nu}{r} \int_{\partial B_{r}} x \times(\omega \times x) \mathrm{d} S=8 \pi \nu r^{3} \omega .
\end{gathered}
$$

We have $u \in L^{q}\left(\Omega_{r}\right)^{3}$ for all $q>3 / 2$ and

$$
\|u\|_{q, \Omega_{r}} \leqslant C(q)|\omega| r^{1+3 / q} .
$$

In particular, $u$ has finite kinetic energy with $\|u\|_{2, \Omega_{r}} \leqslant C|\omega| r^{5 / 2}$. For the derivatives of $u$, we have

$$
\begin{aligned}
& \nabla u \in L^{q}\left(\Omega_{r}\right)^{3 \times 3}, \forall q>1 \text {, with }\|\nabla u\|_{q, \Omega_{r}} \leqslant C(q)|\omega| r^{3 / q}, \\
& D^{2} u \in L^{q}\left(\Omega_{r}\right)^{3 \times 3 \times 3}, \forall q \in\left[1, \infty\left[\text {, with }\left\|D^{2} u\right\|_{q, \Omega_{r}} \leqslant C(q)|\omega| r^{3 / q-1} .\right.\right.
\end{aligned}
$$

\section{References}

[1] Carlos Conca, Jorge San Martín H., and Marius Tucsnak. Existence of solutions for the equations modelling the motion of a rigid body in a viscous fluid. Comm. Partial Differential Equations, 25(5-6):1019-1042, 2000.

[2] Francesca Crispo and Paolo Maremonti. An interpolation inequality in exterior domains. Rend. Sem. Mat. Univ. Padova, 112:11-39, 2004.

[3] Patricio Cumsille and Takéo Takahashi. Wellposedness for the system modelling the motion of a rigid body of arbitrary form in an incompressible viscous fluid. Czechoslovak Math. J., 58(133)(4):961-992, 2008.

[4] Masoumeh Dashti and James C. Robinson. The motion of a fluid-rigid disc system at the zero limit of the rigid disc radius. Arch. Ration. Mech. Anal., 200(1):285-312, 2011.

[5] B. Desjardins and M. J. Esteban. Existence of weak solutions for the motion of rigid bodies in a viscous fluid. Arch. Ration. Mech. Anal., 146(1):59-71, 1999.

[6] B. Desjardins and M. J. Esteban. On weak solutions for fluid-rigid structure interaction: compressible and incompressible models. Comm. Partial Differential Equations, 25(78):1399-1413, 2000.

[7] Eduard Feireisl. On the motion of rigid bodies in a viscous fluid. Appl. Math., 47(6):463-484, 2002. Mathematical theory in fluid mechanics (Paseky, 2001). 
[8] Giovanni P. Galdi. On the motion of a rigid body in a viscous liquid: a mathematical analysis with applications. In Handbook of mathematical fluid dynamics, Vol. I, pages 653-791. North-Holland, Amsterdam, 2002.

[9] Giovanni P. Galdi and Ana L. Silvestre. Strong solutions to the problem of motion of a rigid body in a Navier-Stokes liquid under the action of prescribed forces and torques. In Nonlinear problems in mathematical physics and related topics, I, volume 1 of Int. Math. Ser. (N. Y.), pages 121-144. Kluwer/Plenum, New York, 2002.

[10] G.P. Galdi. Slow motion of a body in a viscous incompressible fluid with application to particle sedimentation. In Recent developments in partial differential equations, pages 1-35. Aracne, Rome, 1998.

[11] G.P. Galdi. On the steady self-propelled motion of a body in a viscous incompressible fluid. Arch. Ration. Mech. Anal., 148(1):53-88, 1999.

[12] Céline Grandmont and Yvon Maday. Existence for an unsteady fluid-structure interaction problem. M2AN Math. Model. Numer. Anal., 34(3):609-636, 2000.

[13] Max D. Gunzburger, Hyung-Chun Lee, and Gregory A. Seregin. Global existence of weak solutions for viscous incompressible flows around a moving rigid body in three dimensions. J. Math. Fluid Mech., 2(3):219-266, 2000.

[14] John G. Heywood. The Navier-Stokes equations: on the existence, regularity and decay of solutions. Indiana Univ. Math. J., 29(5):639-681, 1980.

[15] K.-H. Hoffmann and V. N. Starovoitov. On a motion of a solid body in a viscous fluid. Two-dimensional case. Adv. Math. Sci. Appl., 9(2):633-648, 1999.

[16] D. Iftimie, M. C. Lopes Filho, and H. J. Nussenzveig Lopes. Two-dimensional incompressible viscous flow around a small obstacle. Math. Ann., 336(2):449-489, 2006.

[17] Dragos Iftimie and James P. Kelliher. Remarks on the vanishing obstacle limit for a 3D viscous incompressible fluid. Proc. Amer. Math. Soc., 137(2):685-694, 2009.

[18] N. V. Judakov. The solvability of the problem of the motion of a rigid body in a viscous incompressible fluid. Dinamika Splošn. Sredy, (Vyp. 18 Dinamika Zidkost. so Svobod. Granicami):249-253, 255, 1974.

[19] Horace Lamb. Hydrodynamics. Cambridge Univ. Press, New York, 1932.

[20] Pierre-Louis Lions. Mathematical topics in fluid mechanics. Vol. 1, volume 3 of Oxford Lecture Series in Mathematics and its Applications. The Clarendon Press Oxford University Press, New York, 1996. Incompressible models, Oxford Science Publications.

[21] James C. Robinson. A coupled particle-continuum model: well-posedness and the limit of zero radius. Proc. R. Soc. Lond. Ser. A Math. Phys. Eng. Sci., 460(2045):1311-1334, 2004.

[22] Denis Serre. Chute libre d'un solide dans un fluide visqueux incompressible. Existence. Japan J. Appl. Math., 4(1):99-110, 1987.

[23] Ana Leonor Silvestre. On the slow motion of a self-propelled rigid body in a viscous incompressible fluid. J. Math. Anal. Appl., 274(1):203-227, 2002.

[24] George Gabriel Stokes. On the effect of the internal friction of fluids on the motion of pendulums. Cambridge Philosophical Society, 9:8-106, 1851.

[25] Takéo Takahashi and Marius Tucsnak. Global strong solutions for the two-dimensional motion of an infinite cylinder in a viscous fluid. J. Math. Fluid Mech., 6(1):53-77, 2004.

[26] Roger Temam. Navier-Stokes equations. Theory and numerical analysis. North-Holland Publishing Co., Amsterdam, 1977. Studies in Mathematics and its Applications, Vol. 2.

[27] H. F. Weinberger. On the steady fall of a body in a Navier-Stokes fluid. In Partial differential equations (Proc. Sympos. Pure Math., Vol. XXIII, Univ. California, Berkeley, Calif., 1971), pages 421-439. Amer. Math. Soc., Providence, R. I., 1973. 